246 Dr. Joseph Coats-The Pathology of Diabetes Mellitus.

the fever. Poultices were re-applied for three days, when the abscess burst, discharging about 3 ss of yellow pus. Improvement again set in and steadily continued; so that on 10th July he was allowed to get up and move a little about. A slight hardness could still be felt. He experienced no pain or discomfort from it, and had become stouter than ever. On 10th August T. W. called on me. He reported himself as feeling perfectly well and desired permission to resume work. He felt no pain in the bowels and could walk about as well as ever. On examining the abdomen the lump was gone, only a slight ridge of hardness remained. This was felt for about five inches, running parallel with the bodies of the vertebræ, but a little in front and to the right side of them. On deep and firm pressure over it, a little tenderness was felt, but not what would amount to pain.

As to the cause of abscess arising in this case of fever I can say nothing definite. The time of the illness at which I was called in, to a great extent prevents that. It appears, however, that some eight days previous to taking his bed, he had, while at work, given his back a sudden wrench. This annoyed him for a day or two, but no longer. He thought nothing of it until questioned afterwards. This may have been the cause. Were it so, a continuation of the pain in the back would have been expected. This was not the case. From what, then, could this abscess arise? Possibly from suppuration of some of the mesenteric glands. The situation of the pain (always in the bowels) along with the hard ridge, which was felt on deep pressure over the abdomen, would point to this. Although our patient was considered an extra strong man and always enjoyed good health, his family had suffered more or less from scrofulous diseases of the joints and glands.

Another probable cause of this abscess may be mentioned. Partial peritonitis may have been set up from rupture of the bowel, or otherwise, ending in the formation of abscess.

\title{
THE PATHOLOGY OF DIABETES MELLITUS.
}

BY JOSEPH COATS, M.D.

THE pathology of diabetes is a subject of considerable difficulty, and it may be well at the outset to set forth the problem which requires solution. 
The blood and urine contain normally a small quantity of grape sugar, but in diabetes this undergoes an enormous increase. In the normal condition the sugar is at a constant minimum, unaffected by the amount or kind of food taken. In diabetes there is commonly a very obvious relation between the food taken and the sugar. There are some diabetics who cease to excrete any excess of sugar so long as they abstain from starch or sugar, but whenever they take any such food it is mostly converted into grape sugar and so excreted in the urine. On the other hand there are diabetics who excrete an excess of sugar whatever the kind of food they take, and though the sugar may be diminished by the use of a mainly nitrogenous diet, yet it cannot be thus made to disappear. In that case it is obvious that sugar is formed not only from carbo-hydrates, but also from the albuminous principles of the food. According to Traube diabetes is divisible into a slighter and more severe form according as the sugar is formed from the carbo-hydrates alone or from nitrogenous principles as well, and it is probable that these forms represent an earlier and a later stage in the disease. Donkin, in view of the relation of the sugar production to the food, distinguishes three stages, first that in which only the starch or sugar of the food furnish the grape sugar, second that in which fats as well as these are converted, and third that in which albuminous foods, as well as starchy and oily, undergo conversion into grape sugar.

It is obvious that in this latter stage there is a great consumption of albumen, and in the process of formation of the sugar urea is formed as a waste product in the chemical transformation. We find, therefore, that the urea is also greatly increased in the urine, amounting sometimes to two or three times the normal quantity in the twenty-four hours. In this stage of the disease there is no doubt that the fatty and albuminous constituents of the tissues are also used for the formation of sugar, and that the albuminous principles of the tissues like the albumen of the food furnish sugar and urea which appear in the urine.

The problem which we have to face then is, that in diabetes there is, for some reason or other, an excessive demand for sugar in the organism. The greed for sugar seems to acquire intensity as the disease advances, so that while at first it is only the carbo-hydrates which fall a victim to it, there is ultimately a consumption of the nitrogenous foods and even of the nitrogenous tissues. We have to enquire what may be the meaning of this immense demand for sugar. Look- 
ing to the manner in which the processes of the body are arranged, we may take it for granted that there is some need to be supplied or else such an excess of material would not be furnished.

Sugar is a crystallisable substance, and wherever it is formed it will readily be dissolved by the juices and find its way into the blood. Its occurrence in the blood and its excretion by the urine are therefore simply a result of the ready solubility and diffusibility of the substance. Its presence in these fluids throws no direct light on its place of origin.

It is natural to look to the liver as the source of the sugar. It is well known that the liver is the place of formation of a substance nearly allied to sugar, namely glycogen. This substance, as its name implies, has a great tendency to become converted into sugar. It is true that in the living body the liver, apparently, contains glycogen and not sugar. If the liver of an animal be cut out immediately after death and without delay placed in boiling water after being cut into small pieces, then it will be chiefly glyengen that will be found, and any sugar that exists has probably formed after death by the transformation of the glycogen. It is difficult indeed to avoid the occurrence of traces of sugar in this experiment, and if the removal of the liver be delayed a large amount of sugar will be found. These facts show that glycogen is always just ready to be converted into sugar, its conversion is, as it were, every moment imminent. During life this conversion does not take place to any considerable extent, and it is even doubtful whether the small amount of sugar existing normally in the body is due to a conversion of the glycogen in the liver, a comparison of the blood in the hepatic vein with that in the portal giving doubtful results.

We have to consider, however, whether a pathological conversion of the glycogen into sugar may not occur in the liver, and in this regard the observations of Bock and Hoffman are of great interest. These observers succeeded in the production of an artificial diabetes mellitus by injecting into the blood of rabbits large quantities of a watery solution of common salt. The animals, soon after the injection was made, began to secrete a large quantity of urine, and this urine soon became saccharine. If the injection of salt solution was persisted in, the sugar by and bye diminished in the urine and ultimately disappeared.

The question now is as to the source of the sugar, and the condition of the liver was examined with this view. It became abundantly clear that the sugar in the urine came from the liver and was caused by the conversion of the glycogen into 
sugar. In all cases where the animal was killed after the diabetes had passed off, the liver was found free both of the glycogen which exists normally and of sugar. If, on the other hand, the animal was killed while the mellituria existed, then glycogen and sugar were both present in the liver. The inference from these observations is perfectly obvious. The abnormal condition of the blood causes the transformation of the liver glycogen into sugar, and the latter being a crystalloid and readily diffusible it is at once washed out of the hepatic cells and passes into the circulation and on into the urine. The diabetes ceases because all the glycogen in the liver has undergone conversion into sugar and the source of supply is exhausted, the liver in these cases being found free of both glycogen and sugar. We may say, therefore, that a glycosuria may be produced by the conversion of the glycogen within the liver into sugar.

It may be said that the diabetes thus produced is temporary and not comparable with the permanent disease in the human subject. Let us suppose, however, that diabetes is due to the - conversion of the liver glycogen into sugar, then the problem with which we started will undergo a considerable modification. We saw that there is an excessive production of sugar, but in the view now brought forward this will resolve itself into an excessive production of glycogen which undergoes conversion into sugar: The problem therefore presents itself-Is there anything to account for the excessive formation of glycogen?

It is to be presumed that glycogen has an important function in the animal body. Whether it be used in the production of muscular force, as many suppose, or otherwise, it seems clear that such an organ as the liver would not be engaged in its formation unless it had an important part to play. It is to be presumed then that glycogen supplies some need in the organism and that it is formed in the liver for that purpose.

But if, as soon as it is formed in the liver, it is transformed into sugar then the need in the organism will not be supplied. The sugar being a crystalloid will be washed out, and very little glycogen will get into the blood. In such circumstances the organism will have, as it were, a hunger for glycogen, the liver will be stimulated to supply it, and will produce more glycogen. As soon as it is formed, however, the glycogen will be transformed into sugar and the excessive demand for glycogen will only result in an excessive supply of sugar. In this case the liver will be stimulated to an ever-increasing 
250 Dr. Joseph Coats-The Pathology of Diabetes Mellitus.

formation of glycogen which will immediately pass into sugar. We can understand how in the earlier stages all foods which are near to glycogen in their chemical composition will be at once utilised, and how as the disease goes on and the demand becomes more urgent the other kinds of foods and even the tissues of the body will be used.

So long therefore as the hepatic cells retain their activity we may presume that they will react to the hunger for glycogen, and the production of sugar will be the result. If the hepatic cells be destroyed or weakened the probability is that the diabetes will diminish, the disease requiring that the hepatic cells retain their energy.

It would appear from the above that the diabetes is explainable on the supposition that the glycogen undergoes abnormally a transformation into sugar, and that this occurs in the liver itself. We have now to inquire whether any indication exists as to the cause of this transformation.

In the experiments of Bock and Hoffmann already alluded to it appears that a particular abnormal condition of the blood is capable of producing this transformation. An enormous dilution of the blood with salt solution seems capable of inducing the liver glycogen to pass into sugar. A temporary diabetes has been produced in a variety of other ways and all of them are explicable on the ground of an abnormal condition of the circulation of the liver.

The inhalation of nitrite of amyle induces a temporary glycosuria. We know that this agent produces a general vasomotor paralysis, and a general dilatation of the systemic arteries. With the other arteries those of the abdomen will dilate and the blood passing through the capillaries at an increased rate will reach the portal 'vein at a higher pressure than normal. The circulation in the liver will therefore be accelerated and the blood will be less venous in character, having passed rapidly through the capillaries of the intestine and other organs.

The celebrated diabetic puncture of Bernard is made in the medulla oblongata, and we know that in this part of the nervous system are situated the principal vaso-motor centres of the body. There is therefore here also a paralysis of the arteries just as after the inhalation of nitrite of amyle. Injuries to the brain, spinal cord, and sympathetic sometimes produce a temporary glycosuria, and they also may cause vaso-motor paralysis.

The observations of Pavy are important in this regard. $\mathrm{He}$ produced glycosuria by the injection of defibrinated arterial 
blood into the portal vein, and here again there is an excessive supply of blood to the liver. But then Pavy also produced glycosuria by ligaturing the portal vein, and so cutting off the blood supply except through the hepatic artery, and at first this result seems a very contradictory one.

The effect of that experiment would be to cause the liver to be supplied with arterial blood alone, and we may presume that the hepatic artery would dilate and so allow of an additional flow of blood. According to the researches of Cohnheim and Litten (Virchow's Archiv, vol. lxvii) the blood of the hepatic artery, after supplying the connective tissue, gall ducts, and walls of the large blood-vessels, passes into the inter-lobular veins and on into the proper hepatic capillaries, so that the blood of the hepatic artery is finally distributed with that of the portal vein. When the portal vein is closed, the circulation in the liver will be kept up, but by the blood of the hepatic artery.

From these observations Pavy concluded that the cause of the glycosuria when the portal vein was ligatured was the circulation of arterial blood in the liver, and he proceeded to determine whether it was possible in animals to produce diabetes by supersaturating the whole blood with oxygen, so that the blood in the portal vein would be virtually arterial. $\mathrm{He}$ effected this in various ways, by causing the animals to inhale oxygen; by using artificial respiration till the fact that the animals ceased to make any spontaneous respiratory movements showed a deficiency of carbonic acid; by causing the animals to inhale carbonic oxide. In all these ways glycosuria was produced. It would appear therefore that when diabetes is artificially produced in animals the one essential condition is that the liver should be supplied with unduly oxygenated blood, such blood causing the glycogen to be transformed into sugar in the liver itself.

We have now to consider the question whether this view will explain the occurrence of diabetes in the human subject. Is there any evidence of such an alteration of the circulation in the liver in any cases of diabetes? It is to be noted here that in order to an active hyperæmia of the portal circulation there must be an active hyperæmia in the parts to which the cœliac and mesenteric arteries are distributed. The circulation in the portal vein depends entirely on that in these vessels, and unless the blood passes in greater abundance and more rapidly through the vessels of the abdominal viscera from which the portal blood comes, then it cannot pass more quickly through the portal vein. Moreover, if diabetes depends 
on the blood in the portal vein being unduly oxygenated, this can only happen because it passes so quickly through the capillaries of the alimentary canal and other organs that it is imperfectly deoxygenated. The question to be answered then is, whether there is any evidence of diabetes being due to a vaso-motor paralysis inducing an active hyperæmia of all these organs.

Such a vaso-motor paralysis might arise by disease of the central nervous system, or of the local centres in the abdomen, mainly the cœliac plexus. In regard to the latter, some cases have been recorded (by Ferichs, Klebs, and Recklinghausen) in which diabetes has coexisted with atrophy, formation of calculi, or cancer of the pancreas. In cases observed by Klebs there was, along with the atrophy of the pancreas, a very marked change in the coliac plexus consisting of a great destruction of ganglion cells. It seems very probable considering the nearness of the pancreas to the coeliac plexus that a cancer or the changes resulting from the formation of calculi in the ducts of the pancreas might extend to the cœliac plexus. Klebs accounts for the diabetes in his cases on the view that there was vaso-motor paralysis produced by the destruction of the ganglion cells of the cœliac plexus. In one of his cases indeed there was observed after death an extraordinary dilatation of the hepatic and splenic arteries and the gastric branches of the coeliac axis, the last named branch attaining the size of a goose quill and presenting a highly convoluted course. These facts seem to confirm the view that in man diabetes may be produced by paralysis of the arteries in the domain of the cœliac plexus.

There have been also cases in which diabetes seemed to follow injury to the spinal cord or medulla oblongata, but these have been cases of temporary glycosuria comparable to that following Bernard's diabetic puncture. On the other hand there have been a few instances of softening of the brain and intra-cranial tumours in cases of diabetes, but these have been too rare to be regarded as of much importance in the pathology of diabetes. For the most part there is little or no alteration visible to the naked eye in the central nervous system.

Recently Dickinson has described minute changes in the central nervous system, consisting mainly in excavations around the arteries, produced apparently by exudation from these vessels with disintegration of the brain substance around. In very recent cases there was even accumulation of leucocytes or hæmorrhage around the arteries, but in the older cases a 
cribriform appearance, the arteries being surrounded by widened canals. These lesions are scattered through the brain, the largest and most striking being present in the corpora striata, optic thalami, pons, medulla oblongata, and cerebellum. They are less marked in the cord where in addition there is usually great dilatation of the central canal. The white matter of the cerebral convolutions also frequently presents these lesions.

These lesions are held by Dickinson to be primary and directly related to the cause of the disease. If this view be correct, then we should have lesions of the central nervous system leading to diabetes, and doing so probably by vasomotor paralysis of the arteries of the abdominal viscera. This is open to the objection, however, that these lesions in the nervous system may possibly be secondary, due to a dilatation of the arteries not to exudation from them, especially as we know that exudation occurs rather from capillaries and veins than arteries.

Turning to the abdominal organs themselves, there have been in many cases indications of congestion observed. We have already referred to the enlargement of the arteries in the case described by Klebs. The liver itself is generally described as congested, and in a few cases there has been thrombosis of the portal vein.

The characteristic red tongue of advanced diabetes has been cited by Pavy as evidence of a congestion of the alimentary canal. The congestion only in the later periods extends to the mouth, the vaso-motor paralysis being progressive.

\section{SALICYLIC ACID : ITS HISTORY AND USES.}

\section{By J. Walls White, M.D., Glasgow.}

Although salicylic acid has been known to the profession for several years, there still remains a general want of appreciation of its chemical relations, as also a lack of knowledge as to its medicinal powers. The rapidly extending application of it to surgery and medicine indicates it to be a therapeutic agent of great value. It was first known as far back as the year 1838, when Piria and Ething extracted it from the blossoms of the spirea ulmaria. Since that time several chemists. have prepared it from other vetegable substances, chiefly from the 